TRANSACTIONS OF THE

AMERICAN MATHEMATICAL SOCIETY

Volume 363, Number 11, November 2011, Pages 6143-6158

S 0002-9947(2011)05392-4

Article electronically published on May 4, 2011

\title{
ON GENERALIZED WHITEHEAD PRODUCTS
}

\author{
BRAYTON GRAY
}

\begin{abstract}
We define a symmetric monodical pairing $G \circ H$ among simply connected co-H spaces $G$ and $H$ with the property that $S(G \circ H)$ is equivalent to the smash product $G \wedge H$ as co-H spaces. We further generalize the Whitehead product map to a map $G \circ H \rightarrow G \vee H$ whose mapping cone is the cartesian product.
\end{abstract}

Whitehead products have played an important role in unstable homotopy. They were originally introduced Whi41 as a bilinear pairing of homotopy groups:

$$
\pi_{m}(X) \otimes \pi_{n}(X) \rightarrow \pi_{m+n-1}(X), \quad m, n>1 .
$$

This was generalized ([Ark62, Coh57, Hil59]) by constructing a map

$$
\mathrm{W}: S(A \wedge B) \rightarrow S A \vee S B \text {. }
$$

Precomposition with $\mathrm{W}$ defines a function on based homotopy classes:

$$
[S A, X] \times[S B, X] \rightarrow[S(A \wedge B), X]
$$

which is bilinear in case $A$ and $B$ are suspensions.

The case where $A$ and $B$ are Moore spaces was central to the work of Cohen, Moore and Neisendorfer ([CMN79]). In [Ani93] and in particular [AG95, this work was generalized. Much of this has since been simplified in GT10, but further understanding will require a generalization from suspensions to co- $\mathrm{H}$ spaces.

The purpose of this work is to carry out and study such a generalization. Let $\mathcal{C O}$ be the category of simply connected co-H spaces and co-H maps. We define a functor

$$
\begin{aligned}
& \mathcal{C O} \times \mathcal{C O} \rightarrow \mathcal{C O}, \\
& (G, H) \rightarrow G \circ H,
\end{aligned}
$$

and a natural transformation

$$
\mathrm{W}: G \circ H \rightarrow G \vee H
$$

generalizing the Whitehead product map. The existence of $G \circ H$ generalizes a result of Theriault [The03] who showed that the smash product of two simply connected co-associative co-H spaces is the suspension of a co-H space. We do not need the co$\mathrm{H}$ spaces to be co-associative and require only one of them to be simply connected 1 We call $G \circ H$ the Theriault product of $G$ and $H$.

Received by the editors November 28, 2009 and, in revised form, June 8, 2010.

2010 Mathematics Subject Classification. Primary 55P99, 55Q15, 55Q20, 55Q25.

${ }^{1}$ In fact we can define $G \circ H$ for any two co-H spaces but require at least one of them to be either simply connected or a suspension in order to obtain the co-H space structure map on $G \circ H$. Recently Grbić, Theriault and Wu have shown that the smash product of any two co-H spaces is the suspension of a co-H space, but their construction cannot satisfy Theorem [1a) below [GTW].

C) 2011 American Mathematical Society
Reverts to public domain 28 years from publication 6143 
We summarize our results in the following theorems.

Theorem 1. There is a functor $\mathcal{C O} \times \mathcal{C O} \rightarrow \mathcal{C O}$ given by

$$
(G, H) \rightarrow G \circ H
$$

and equivalences in $\mathcal{C O}$ :

(a) $(S X) \circ H \simeq X \wedge H$,

(b) $S(G \circ H) \simeq G \wedge H$,

(c) $\left(G_{1} \vee G_{2}\right) \circ H \simeq G_{1} \circ H \vee G_{2} \circ H$

and homotopy equivalences:

(d) $G \circ H \simeq H \circ G$,

(e) $(G \circ H) \circ K \simeq G \circ(H \circ K)$.

Theorem 2. There is a natural transformation

$$
\mathrm{W}: G \circ H \rightarrow G \vee H
$$

which is the Whitehead product map in case $G$ and $H$ are both suspensions. Furthermore, there is a homotopy equivalence

$$
G \times H \simeq G \vee H \cup_{\mathrm{W}} C(G \circ H) .
$$

The next theorem concerns the inclusion of the fiber in certain standard fibration sequences Gra71]:

$$
\begin{gathered}
G \rtimes \Omega H \stackrel{\iota_{1}}{\rightarrow} G \vee H \stackrel{\pi_{2}}{\longrightarrow} H, \\
\Omega G * \Omega H \stackrel{\iota_{2}}{\longrightarrow} G \vee H \rightarrow G \times H .
\end{gathered}
$$

Define $\operatorname{ad}^{n}(H)(G)$ inductively by $\operatorname{ad}^{0}(H)(G)=G$ and

$$
\operatorname{ad}^{n}(H)(G)=\left[a d^{n-1}(H)(G)\right] \circ H
$$

and by an iterated Whitehead product

$$
a d^{n}: a d^{n}(H)(G) \rightarrow G \vee H
$$

as the composition

$$
a d^{n}(H)(G) \stackrel{\mathrm{W}}{\longrightarrow} a d^{n-1}(H)(G) \vee H \stackrel{a d^{n-1} \vee 1}{\longrightarrow} G \vee H \vee H \rightarrow G \vee H .
$$

Theorem 3. Suppose $G$ and $H$ are simply connected co-H spaces. Then there are homotopy equivalences:

(a) $G \rtimes \Omega H \simeq \bigvee_{n \geqslant 0} a d^{n}(H)(G)$, where $\iota_{1}$ corresponds to $a d^{n}$ on the appropriate factor,

(b) $\Omega G * \Omega H \simeq \bigvee_{\substack{i \geqslant 0 \\ j \geqslant 1}} a d^{j}(H)\left(a d^{i}(G)(G)\right)$, where $\iota_{2}$ corresponds to $a d^{j}\left(a d^{i}\right)$ on to the appropriate factor,

(c) $S \Omega G \simeq \bigvee_{n \geqslant 0} a d^{n}(G)(G)$, where the composition $S \Omega G \rightarrow S \Omega G \vee S \Omega G \rightarrow$ $G \vee G$ corresponds to the appropriate iterated Whitehead product on each factor. 
It should be pointed out that equivalence (c) generalizes the result of Theriault The03, 1.1] where it is shown that a simply connected co-associative co-H space decomposes

$$
\Sigma \Omega G \simeq \bigvee_{n \geqslant 1} M_{n}
$$

for some spaces $M_{n}$, which are not further decomposed.

Theorem 4. Suppose $X$ is finite dimensional and $f: S X \rightarrow G \vee H$. Then $f$ is the sum of the projections onto $G$ and $H$ and a finite sum of iterated Whitehead products.

Throughout this work we will assume that all spaces are of the homotopy type of a CW complex. All homology and cohomology will be with a field of coefficients. We will often show that a map between simply connected CW complexes is a homotopy equivalence by showing that it induces an isomorphism in homology with an arbitrary field of coefficients, without further comment.

Section „will be devoted to some general remarks about telescopes, and we will construct the Theriault product in section . Theorem 1 will follow from Propositions 2.3, 2.5 and 2.7. The functor in Theorem 2 is defined after Corollary 3.2, and the equivalence follows from Proposition 3.8 . The proof of the first part of Theorem 3 occurs just prior to Corollary 3.5 and the rest occurs following Corollary 3.5 . Theorem 4 follows from Corollary 3.7

\section{SECTION 1}

In this section we will discuss some general properties of telescopes of a self map $e: G \rightarrow G$, where $G$ is a co-H space. We do not assume that $e$ is idempotent. We will call $e$ quasi-idempotent if the induced homomorphism in homology satisfies the equation

$$
\left(e_{*}\right)^{2}=-e_{*},
$$

where $u$ is a unit. We construct two telescopes:

$$
\begin{gathered}
T(e): G \stackrel{e}{\rightarrow} G \stackrel{e}{\rightarrow} G \rightarrow \ldots, \\
T(1+e): G \stackrel{1+e}{\longrightarrow} G \stackrel{1+e}{\longrightarrow} G \rightarrow \ldots
\end{gathered}
$$

and a map:

$$
\Gamma: G \rightarrow G \vee G \stackrel{\Gamma_{1} \vee \Gamma_{2}}{\longrightarrow} T(e) \vee T(1+e) .
$$

Proposition 1.1. If $G$ is simply connected and $e$ is a quasi-idempotent, $\Gamma$ is a homotopy equivalence. Furthermore $H_{*}(T(e))=i m e_{*}$ and $\widetilde{H}_{*}(T(1+e)) \cong \operatorname{ker} e_{*}$.

Proof. Suppose $\Gamma_{*}(\xi)=0$. Since $\left(\Gamma_{1}\right)_{*}(\xi)=0\left(e_{*}\right)^{k}(\xi)=0$ for some $k, e_{*}(\xi)=0$. Since $\left(\Gamma_{2}\right)_{*}(\xi)=0,(1+e)_{*}{ }^{k}(\xi)=0$. However, $(1+e)_{*}^{2}=(1+e)_{*}$, so $\xi=-e_{*}(\xi)=0$. Clearly $\Gamma_{*}$ is onto. Moreover, $H_{*}(T(e)) \cong i m e_{*}$ and $\widetilde{H}_{*}(T(1+e)) \cong i m\left(1+e_{*}\right)=$ ker $e_{*}$.

Corollary 1.2. Suppose $G$ is a simply connected co- $H$ space and $A \subset G$ is a retract of $G$. Let e be the composition

$$
G \stackrel{r}{\rightarrow} A \stackrel{i}{\rightarrow} G
$$


Then $T(e) \simeq A, T(1-e) \simeq G / A$, and the identity map of $T(e)$ can be factored:

$$
T(e) \stackrel{\xi}{\rightarrow} A \stackrel{i}{\rightarrow} G \stackrel{r}{\rightarrow} A \stackrel{\eta}{\rightarrow} T(e),
$$

where $\xi$ and $\eta$ are inverse homotopy equivalences.

Proof. The telescope $T(e)$ and $A$ are both simply connected, and there are maps $T(e) \rightarrow A$ and $A \rightarrow T(e)$ making $A$ a retract of $T(\xi)$; these maps are homotopy equivalences. By the Van Kampen theorem $G / A \simeq G \cup C A$ is simply connected. Since $1-e: G \rightarrow G$ factors through the projection $\pi: G \rightarrow G / A$, we can factor the identity map up to homotopy,

$$
G \rightarrow G \vee G \stackrel{r \vee \pi}{\longrightarrow} A \vee G / A \rightarrow G,
$$

and hence $G \simeq A \vee G / A$. The factorization of the identity map of $T(e)$ is obtained by replacing each space by a telescope where the three in the center are constant.

Now consider two maps $f_{1}, f_{2}: X \rightarrow X$.

Proposition 1.3. $T\left(f_{1} f_{2}\right) \simeq T\left(f_{2} f_{1}\right)$.

Proof. We define maps between the telescopes:

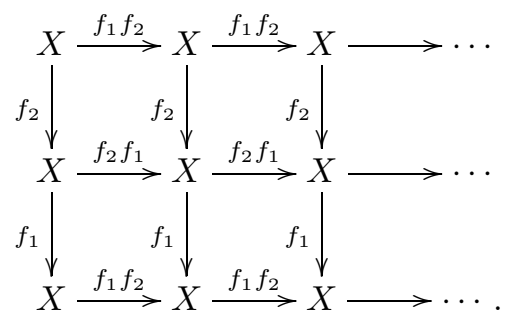

The composition is the shift map which is a homotopy equivalence.

\section{SECTION 2}

In this section we will consider a pair of co-H spaces in which at least one is simply connected. Let $G$ and $H$ be two such co-H spaces with their structure determined by maps $\nu_{1}: G \rightarrow S \Omega G$ and $\nu_{2}: H \rightarrow S \Omega H$, each of which is a right inverse to the respective evaluation maps (see [Gan70]), which we label as $\epsilon_{1}, \epsilon_{2}$. We define self maps of $S(\Omega G \wedge \Omega H)$ as follows:

$$
\begin{aligned}
& e_{1}: S(\Omega G \wedge \Omega H) \stackrel{\epsilon_{1} \wedge 1}{\longrightarrow} G \wedge \Omega H \stackrel{\nu_{1} \wedge 1}{\longrightarrow} S(\Omega G \wedge \Omega H), \\
& e_{2}: S(\Omega G \wedge \Omega H) \stackrel{1 \wedge \epsilon_{2}}{\longrightarrow} \Omega G \wedge H \stackrel{1 \wedge \nu_{2}}{\longrightarrow} S(\Omega G \wedge \Omega H) .
\end{aligned}
$$

Here we freely move the suspension coordinate to wherever it is needed. Clearly $e_{1}$ and $e_{2}$ are idempotents but $e_{1} e_{2}$ is not an idempotent; however it is a quasiidempotent. To see this we need to pay careful attention to the order of the suspension coordinates. Let

$$
T: S^{1} \wedge S^{1} \wedge \Omega G \wedge \Omega H \rightarrow S^{1} \wedge S^{1} \wedge \Omega G \wedge \Omega H
$$

be the map switching the suspension coordinates. What we will show is that the composition

$$
e=\left(S e_{2}\right) \circ T \circ\left(S e_{1}\right)
$$

is an idempotent. Since $T \sim(-1)$ and $\left(S e_{1}\right)$ is a suspension,

$$
e \sim-\left(S e_{2}\right) \circ\left(S e_{1}\right) \text {. }
$$


Once we see that $e$ is an idempotent it follows that $\left(S e_{1}\right) \circ\left(S e_{2}\right)$ is a quasiidempotent.

Now we factor $e$ and note that it contains the composition

$$
S\left(1 \wedge \epsilon_{2}\right) \circ T \circ S\left(\nu_{1} \wedge 1\right) .
$$

However, this composition is equal (no homotopies) to the composition

$$
S G \wedge \Omega H \stackrel{1 \wedge \epsilon_{2}}{\longrightarrow} G \wedge H \stackrel{\nu_{1} \wedge 1}{\longrightarrow} S \Omega G \wedge H .
$$

Consequently, $e$ is equal to the composition

$$
\begin{aligned}
S^{1} \wedge S^{1} \wedge \Omega G \Omega H \simeq S \Omega G \wedge S \Omega H & \stackrel{\epsilon_{1} \wedge \epsilon_{2}}{\longrightarrow} G \wedge H \\
& \stackrel{\nu_{1} \wedge \nu_{2}}{\longrightarrow} S \Omega G \wedge S \Omega H \simeq S^{1} \wedge S^{1} \wedge \Omega G \wedge \Omega H,
\end{aligned}
$$

and this map is clearly an idempotent.

Now assuming that one of $G, H$ is simply connected, it follows that $\Omega G \wedge \Omega H$ is connected, so $S(\Omega G \wedge \Omega H)$ is simply connected. Consequently,

Proposition 2.1. If one of $G$ and $H$ is simply connected, there is a homotopy equivalence,

$$
S(\Omega G \wedge \Omega H) \simeq T\left(e_{1} e_{2}\right) \vee T\left(1+e_{1} e_{2}\right)
$$

Let

$$
\theta: S(\Omega G \wedge \Omega H) \rightarrow T\left(e_{1} e_{2}\right)
$$

be the projection and

$$
\psi: T\left(e_{1} e_{2}\right) \rightarrow S(\Omega G \wedge \Omega H)
$$

be the unique right inverse to $\theta$ which projects trivially onto $T\left(1+e_{1} e_{2}\right)$. These maps determine a co-H space structure on $T\left(e_{1} e_{2}\right)$.

Definition 2.2. $G \circ H=T\left(e_{1} e_{2}\right)$.

Proposition 2.3. Given co- $H$ maps $f: G \rightarrow G^{\prime}$ and $g: H \rightarrow H^{\prime}$, there is an induced co-H map

$$
f \circ g: G \circ H \rightarrow G^{\prime} \circ H^{\prime}
$$

making $G \circ H$ a functor of two variables. In addition there are equivalences of co- $H$ spaces:

(a) $S X \circ H \simeq X \wedge H$,

(b) $S(G \circ H) \simeq G \wedge H$,

(c) $S^{1} \circ H \simeq H$,

and there is a homotopy equivalence $G \circ H \simeq H \circ G$.

Proof. Since $f$ and $g$ are co-H maps, the squares
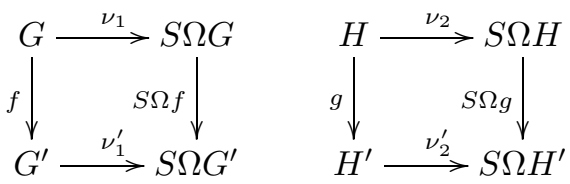

commute up to homotopy. It follows that $f$ and $g$ induce maps that commute with $e_{1}$ and $e_{2}$ and hence with the equivalences of Proposition 2.1, $\theta$ and $\psi$. For part 
(a), observe that the composition $e_{2} e_{1}$ factors

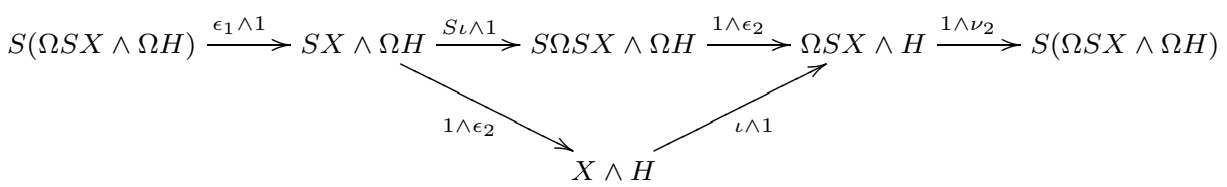

where $\left(1 \wedge \epsilon_{2}\right)\left(\epsilon_{1} \wedge 1\right)$ is a right universe to $\left(1 \wedge \nu_{2}\right)(\iota \wedge 1)$. Thus we can apply Corollary 1.2 to see that $S X \circ H \simeq X \wedge H$ with co-H structure given by the composite $\left(1 \wedge \nu_{2}\right)(\iota \wedge 1)$. This is precisely the co-H structure induced by $\nu_{2}$. Part (b) follows since $S(G \circ H)$ is the telescope of $\bar{e}$ with co-H structure given by $\nu_{1} \wedge \nu_{2}$. Part (c) is a special case of part (a): The last statement follows directly from Proposition 1.3.

To complete the proof of Theorem 1 it remains to prove parts (c), (d), and (e) by Proposition 2.3. Part (d) follows directly from Proposition 1.3. For the other two parts, it will be convenient to describe an alternative definition of $G \circ H$. For this we assume that $G$ is a retract of a space $S X$ and $H$ is a retract of $S Y$ :

$$
G \stackrel{\nu_{1}}{\longrightarrow} S X \stackrel{\epsilon_{1}}{\longrightarrow} G \quad H \stackrel{\nu_{2}}{\longrightarrow} S Y \stackrel{\epsilon_{2}}{\longrightarrow} H .
$$

We can then replace the telescope in the definition by the telescope of the composition:

$$
T: S X \wedge Y \stackrel{1 \wedge \epsilon_{2}}{\longrightarrow} X \wedge H \stackrel{1 \wedge \nu_{2}}{\longrightarrow} S X \wedge Y \stackrel{\epsilon_{1} \wedge 1}{\longrightarrow} G \wedge Y \stackrel{\nu_{1} \wedge 1}{\longrightarrow} S X \wedge Y .
$$

The co-H structures defined by these maps are equivalent to the structures defined by

$$
\widetilde{\nu_{2}}: G \stackrel{\nu_{1}}{\longrightarrow} S X \stackrel{S \widetilde{\epsilon}_{1}}{\longrightarrow} S \Omega G, \quad \widetilde{\nu}_{1}: H \stackrel{\nu_{2}}{\longrightarrow} S Y \stackrel{S \Omega \widetilde{\epsilon}_{2}}{\longrightarrow} S \Omega H,
$$

and we have a homotopy commutative ladder:

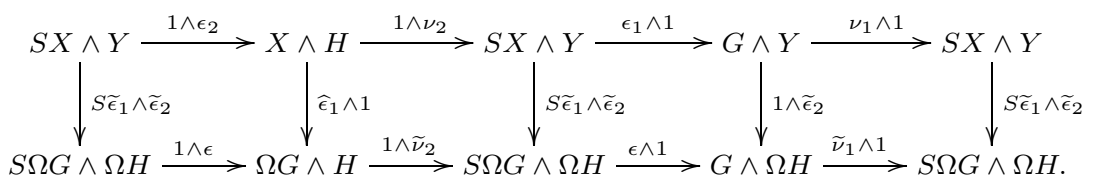

Hence we have a commutative diagram:

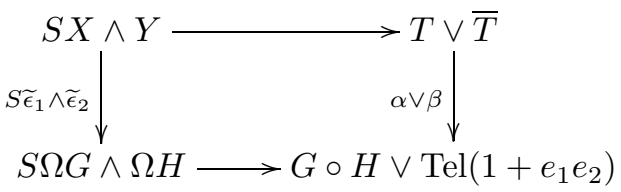

where $\bar{T}$ is the telescope defined by $1+\left(\nu_{1} \wedge 1\right)\left(\epsilon_{1} \wedge 1\right)\left(1 \wedge \nu_{2}\right)\left(1 \wedge \epsilon_{2}\right)$. The map $\alpha: T \rightarrow G \circ H$ is compatible with the homotopy equivalence $S T \simeq G \wedge H \simeq S(G \circ H)$, so is itself a homotopy equivalence. Now choose a right homotopy inverse for the map $S X \wedge Y \rightarrow T$ which projects trivially to $\bar{T}$. Its composite with $S \widetilde{\epsilon}_{1} \wedge \widetilde{\epsilon}_{2}$ will then project trivially to $T\left(1+e_{1} e_{2}\right)$. Hence we have a homotopy commutative diagram:

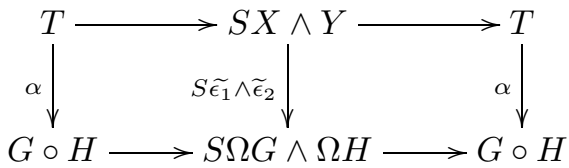

and consequently the co- $\mathrm{H}$ structure on $T$ is compatible under $\alpha$ with the co- $\mathrm{H}$ structure on $G \circ H$. We have proven the proposition. 
Proposition 2.4. Suppose $G$ is represented as a retract of $S X$ and $H$ as a retract of $S Y$. Then $G \circ H$ is homotopy equivalent to the telescope $T$ of the composition

$$
S X \wedge Y \rightarrow G \wedge Y \rightarrow S X \wedge Y \rightarrow X \wedge H \rightarrow S X \wedge Y
$$

as co-H spaces, where the co-H structure on $T$ is given by the equivalence $S X \wedge Y \simeq$ $T \vee \bar{T}$.

Proposition 2.5. $\left(G_{1} \vee G_{2}\right) \circ H \simeq G_{1} \circ H \vee G_{2} \circ H$ as co- $H$ spaces.

Proof. Write $G_{i}$ as a retract of $S X_{i}, i=1,2$. Then $G_{1} \vee G_{2}$ is a retract of $S\left(X_{1} \vee X_{2}\right)$. Thus the telescope for $\left(G_{1} \vee G_{2}\right) \circ H$ is at each point the wedge of the telescopes for $G_{1} \circ H$ and $G_{2} \circ H$.

At this point we will apply Proposition 2.4 to prove Theorem 1(e), the associativity formula. We will make repeated use of

Lemma 2.6. There is a homotopy commutative square

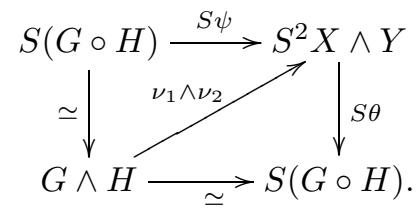

Proof. $G \wedge H$ is a retract of $S^{2} X \wedge Y$, so we may apply Corollary 1.2 ,

Proposition 2.7. There is a homotopy equivalence 2

$$
(G \circ H) \circ K \simeq G \circ(H \circ K) .
$$

Proof. We suppose that $G, H$, and $K$ are presented by retractions

$$
\begin{aligned}
& G \stackrel{\psi_{1}}{\longrightarrow} S X \stackrel{\theta_{1}}{\longrightarrow} G, \\
& H \stackrel{\psi_{2}}{\longrightarrow} S Y \stackrel{\theta_{2}}{\longrightarrow} H, \\
& K \stackrel{\psi_{3}}{\longrightarrow} S Z \stackrel{\theta_{3}}{\longrightarrow} K,
\end{aligned}
$$

and we then construct retractions for $G \circ H$ and $H \circ K$ :

$$
\begin{aligned}
& G \circ H \stackrel{\psi_{3}}{\longrightarrow} S X \wedge Y \stackrel{\theta_{3}}{\longrightarrow} G \circ H, \\
& H \circ K \stackrel{\psi_{4}}{\longrightarrow} S Y \wedge Z \stackrel{\theta_{4}}{\longrightarrow} H \circ K .
\end{aligned}
$$

Using these we construct retractions for $G \circ(H \circ K)$ and $(G \circ H) \circ K$ :

$$
\begin{gathered}
(G \circ H) \circ K \stackrel{\psi}{\rightarrow} S(X \wedge Y \wedge Z) \stackrel{\theta}{\rightarrow}(G \circ H) \circ K, \\
G \circ(H \circ K) \stackrel{\psi^{\prime}}{\longrightarrow} S(X \wedge Y \wedge Z) \stackrel{\theta^{\prime}}{\rightarrow}(G \circ(H \circ K)) .
\end{gathered}
$$

\footnotetext{
${ }^{2}$ Note that we are not asserting a co-H equivalence here and in (c) of Theorem 1 . However, this holds if one of the spaces involved is co-associative. If, for example, $H$ is co-associative, the map$$
G \circ H \rightarrow G \circ S \Omega H \simeq G \wedge \Omega H
$$

is a co-H map by Proposition 2.3. Since this map also has a left homotopy inverse, the co-H structure is determined by that on $G$. 
We will show that $\theta^{\prime} \psi:(G \circ H) \circ K \rightarrow G \circ(H \circ K)$ is a homotopy equivalence. By Lemma 2.6 we have homotopy commutative diagrams:

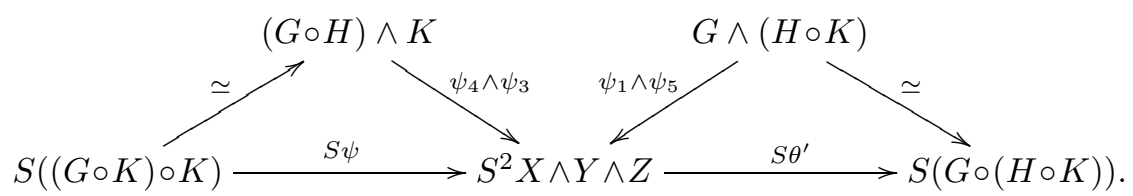

Suspending and applying Lemma 2.6 again, we obtain a homotopy commutative diagram:

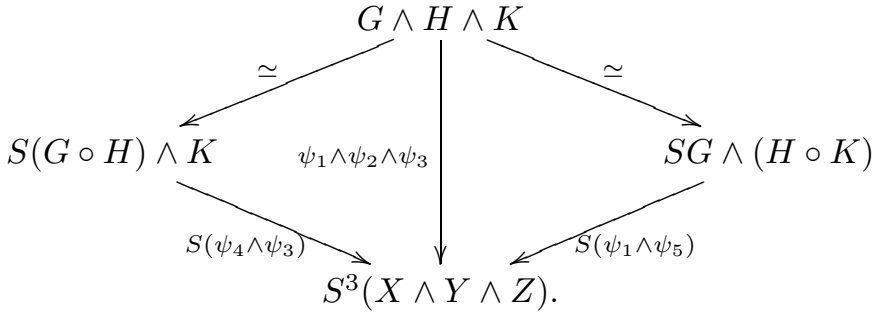

From these diagrams it follows that $S^{2}\left(\theta^{\prime} \psi\right)$ is a homotopy equivalence and hence $\theta^{\prime} \psi$ is as well.

\section{SECTION 3}

In this section we generalize the clutching construction Gra88, Proposition 1] for fibrations over a suspension to fibrations over a co-H space. This allows for the decomposition results in Theorems 2 and 3 .

Proposition 3.1. Suppose $F \rightarrow E \rightarrow G$ is a fibration where $G$ is a co-H space. Then $E / F \simeq G \rtimes F$.

Proof. In the case $G=S X$, we have by [Gra88, Proposition 1]

$$
E \simeq F \cup_{\theta}(C X) \times F .
$$

So $E / F \simeq S X \rtimes F$. It is easy to construct a map $G \rtimes F \rightarrow E / F$ in general. Consider the sequence of pull backs:

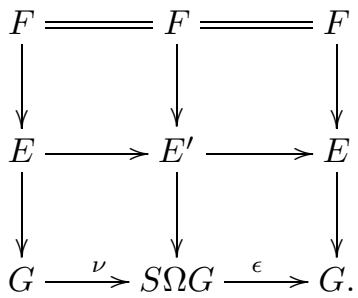

Then we consider the composite

$$
E / F \rightarrow E^{\prime} / F \simeq S \Omega G \rtimes F \rightarrow G \rtimes F,
$$

where the middle equivalence follows since the base is a suspension. Showing that the composite is a homotopy equivalence will take some work.

Since $\nu \epsilon: S \Omega G \rightarrow S \Omega G$ is an idempotent, we can decompose $S \Omega G$ :

$$
S \Omega G \simeq G \vee G^{\prime} .
$$


We now observe that we can construct a quasi-fibration model for a fibration over a one point union. Suppose we have such a fibration

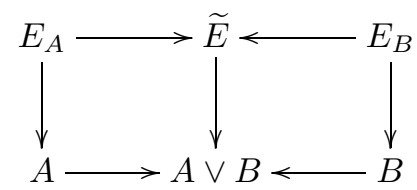

with pull backs $E_{A}$ and $E_{B}$ and fiber $F$. Then we can construct

$$
E_{A} \cup_{F} E_{B},
$$

the union of $E_{A}$ and $E_{B}$ with the subspace $F$ identified. Then

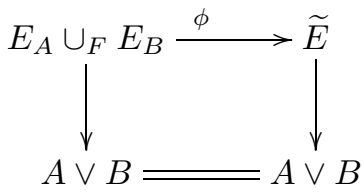

$\phi$ is a homotopy equivalence. In our case $S \Omega G \simeq G \vee G^{\prime}$ and $E_{G}=E, E_{G^{\prime}}=G^{\prime} \times F$, so

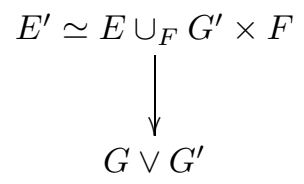

is a quasi-fibering by [DT58, 2.10]. On the other hand $E^{\prime} / F \simeq S \Omega G \rtimes F \simeq$ $G \rtimes F \vee G^{\prime} \rtimes F$, while $E \cup_{F} G^{\prime} \rtimes F \simeq E / F \vee G^{\prime} \times F$. Since the map between $E^{\prime}$ and $E \cup_{F} G^{\prime} \rtimes F$ is a map over $G \vee G^{\prime}$, we see that $E / F \simeq G \times F$.

Corollary 3.2. $S \Omega G \simeq G \rtimes \Omega G$.

Proof. Apply Proposition 3.1 to the path space fibration over $G$.

Proof of Theorem 2. Construction: We now describe our generalization of the Whitehead product. Suppose $G$ and $H$ are co-H spaces and one of them is simply connected. The Whitehead product

$$
\mathrm{W}: G \circ H \rightarrow G \vee H
$$

is then defined as the composition

$$
G \circ H \stackrel{\psi}{\rightarrow} S \Omega G \wedge \Omega H \simeq \Omega G * \Omega H \stackrel{\omega}{\rightarrow} G \vee H,
$$

where $\omega$ is the inclusion of the fiber in the fibration sequence

$$
\Omega G * \Omega H \stackrel{\omega}{\rightarrow} G \vee H \rightarrow G \times H .
$$

Clearly $\psi$ and $\omega$ are natural transformations, so $\mathrm{W}$ is as well.

Before we prove the homotopy equivalence in Theorem 2, we need to establish some results in Theorem 3 . We begin by constructing maps

$$
a d^{n}: a d^{n}(H)(G) \rightarrow G \vee H
$$


inductively. For $n=0$ this is just the inclusion of $G$ in $G \vee H$. For $n>0$ we define $a d^{n}$ as the composition

$$
\begin{aligned}
a d^{n}(H)(G)=\left(a d^{n-1}(H)(G)\right) & \circ H \\
& \rightarrow\left(a d^{n-1}(H)(G)\right) \vee H \rightarrow(G \vee H) \vee H=G \vee H .
\end{aligned}
$$

Next we calculate the effect of $a d^{n}$ in loop space homology:

$$
\Omega\left(a d^{n}\right)_{*}: H_{*}\left(\Omega\left(a d^{n}(H)(G)\right)\right) \rightarrow H_{*}(\Omega(G \vee H)) .
$$

To do this we need some notation. For each co-H space $G$, write

$$
\sigma^{-1}: \widetilde{H}_{r}(G) \rightarrow \widetilde{H}_{r-1}(\Omega G)
$$

for the composition

$$
\widetilde{H}_{r}(G) \stackrel{\nu_{+}}{\longrightarrow} \widetilde{H}_{r}(S \Omega G) \simeq \widetilde{H}_{r-1}(\Omega G) .
$$

Let $\left\{x_{i}\right\}$ be a basis for $\widetilde{H}_{*}(G)$. Then $H_{*}(\Omega G)$ is the tensor algebra on the classes $\left\{\sigma^{-1}\left(x_{i}\right)\right\}$. Given two classes $x \in \widetilde{H}_{r}(G), y \in \widehat{H}_{s}(H)$, we will write

$$
x \circ y \in \widetilde{H}_{r+s-1}(G \circ H)
$$

for the class that corresponds to $x \wedge y \in \widetilde{H}_{r+s}(G \wedge H)$ under the isomorphism

$$
\widetilde{H}_{r-1}(G \circ H) \cong \widetilde{H}_{r}(S(G \circ H)) \simeq \widetilde{H}_{r}(G \wedge H) .
$$

Then the classes $\left\{x_{i} \circ y_{j}\right\}$ form a basis for $\widetilde{H}_{*}(G \circ H)$, where $\left\{x_{i}\right\}$ and $\left\{y_{i}\right\}$, respectively, are bases for $\widetilde{H}_{*}(G)$ and $\widetilde{H}_{*}(H)$.

Proposition 3.3. $(\Omega W)_{*}\left(\sigma^{-1}(x \circ y)\right)= \pm\left[\sigma^{-1} x, \sigma^{-1} y\right]$, where

$$
(\Omega W)_{*}: H_{*}(\Omega(G \circ H)) \rightarrow H_{*}(\Omega(G \vee H)) .
$$

Proof. By Lemma 2.6

$$
\psi_{*}(x \circ y)=e \wedge \sigma^{-1}(x) \wedge \sigma^{-1}(y) \in H_{*}(S \Omega G \wedge \Omega H),
$$

so

$$
(\Omega \psi)_{*}\left(\sigma^{-1}(x \circ y)\right)=\sigma^{-1}(x) \wedge \sigma^{-1}(y) \in H_{*}(\Omega G \wedge \Omega H),
$$

regarded as a submodule of $H_{*}(S \Omega G \wedge \Omega H)$. It now suffices to evaluate the composition

$$
\Omega G \wedge \Omega H \rightarrow \Omega(S \Omega G \wedge \Omega H) \stackrel{\Omega \xi}{\longrightarrow} \Omega(\Omega G * \Omega H) \rightarrow \Omega(G \vee H),
$$

where $\xi$ is the standard homotopy equivalence $S X \wedge Y \simeq X * Y$.

Lemma 3.4. The composition

$$
\Omega G \wedge \Omega H \rightarrow \Omega S(\Omega G \wedge \Omega H) \stackrel{\Omega \xi}{\longrightarrow} \Omega(\Omega G * \Omega H) \rightarrow \Omega(G \vee H)
$$

carries $\sigma^{-1}(x) \wedge \sigma^{-1}(y) \in H_{*}(\Omega G \wedge \Omega H)$ to $\pm\left[\sigma^{-1}(x), \sigma^{-1}(y)\right]$.

Proof. We first need to describe the homotopy equivalence

$$
S X \wedge Y \stackrel{\xi}{\rightarrow} X * Y .
$$


Here we write points of the join as $t x+(1-t) y, 0 \leq t \leq 1$. Therefore, $X * Y$ is the quotient of $X \times I \times Y$ given by the identifications $(x, 0, y) \sim\left(x^{\prime}, 0, y\right)$ and $(x, 1, y) \sim\left(x, 1, y^{\prime}\right)$. Then $\xi$ is given by the formula

$$
\xi(t, x, y)= \begin{cases}(*, 1-3 t, y), & 0 \leq 3 t \leq 1 \\ (x, 3 t-1, y), & 1 \leq 3 t \leq 2 \\ (x, 3-3 t, *), & 2 \leq 3 t \leq 3\end{cases}
$$

The map $\Omega X * \Omega Y \stackrel{\omega}{\rightarrow} X \vee Y$ is given by

$$
\left(\omega_{1}, t, \omega_{2}\right) \rightarrow \begin{cases}\omega_{1}(2 t), & 0 \leq 2 t \leq 1 \\ \omega_{2}(2-2 t), & 1 \leq 2 t \leq 2\end{cases}
$$

Combining these we get

$$
S \Omega X * \Omega Y \stackrel{\xi}{\rightarrow} \Omega X * \Omega Y \stackrel{\omega}{\rightarrow} X \vee Y
$$

with a 6-part formula:

$$
\left(t, \omega_{1}, \omega_{2}\right)= \begin{cases}\left(*, \omega_{2}(6 t)\right), & 0 \leq 6 t \leq 1 \\ *, & 1 \leq 6 t \leq 2 \\ \left(\omega_{1}(6 t-2), *\right), & 2 \leq 6 t \leq 3 \\ \left(*, \omega_{2}(4-6 t)\right), & 3 \leq 6 t \leq 4 \\ *, & 4 \leq 6 t \leq 5 \\ \left(\omega_{1}(6-6 t), *\right), & 5 \leq 6 t \leq 6\end{cases}
$$

so the adjoint takes the pair $\left(\omega_{1}, \omega_{2}\right)$ to the product of loops $\omega_{1}^{-1} \omega_{2}^{-1} \omega_{1} \omega_{2}$. The effect of this on a primitive element is the graded commutator.

Now the iterated circle product $a d^{n}(H)(G)$ has homology generated by classes of the form

$$
\left(\cdots\left(\left(x \circ y_{1}\right) \circ y_{2}\right) \cdots \circ y_{n}\right)
$$

where $x \in \widetilde{H}_{*}(G)$ and $y_{i} \in \widehat{H}_{*}(H)$. By Proposition 3.3.

$$
(\Omega W)_{*}\left(\sigma^{-1}\left(\cdots\left(x \circ y_{1}\right) \circ y_{2}\right) \cdots \circ y_{n}\right)
$$

is \pm the graded commutator

$$
\left[\cdots\left[\left[\sigma^{-1}(x), \sigma^{-1}\left(y_{1}\right)\right], \sigma^{-1}\left(y_{2}\right)\right] \cdots \sigma^{-1}\left(y_{*}\right)\right],
$$

where the classes $x$ and $y_{i}$ are thought of as classes in $\widetilde{H}_{*}(G \vee H)$.

Proof of Theorem $3(\mathrm{a})$. Now let $G_{*}=\widetilde{H}_{*}(G)$ and $H_{*}=\widetilde{H}_{*}(H)$. Let $L\left(G_{*} \oplus H_{*}\right)$ be the free Lie algebra generated by $G_{*}$ and $H_{*}$, and let $L\left(H_{*}\right)$ be the free Lie algebra generated by $H_{*}$. Then Neisendorfer has analyzed the kernel

$$
L\left(G_{*} \vee H_{*}\right) \rightarrow L\left(H_{*}\right)
$$

([Nei09, 8.7.4]). He has shown that this is the free Lie algebra

$$
L\left(\bigoplus_{n \geqslant 0} a d^{n}\left(H_{*}\right)\left(G_{*}\right)\right) .
$$


The universal enveloping algebra is thus the tensor algebra generated by the elements $a d^{n}\left(H_{*}\right)\left(G_{*}\right)$ for $n \geqslant 0$. Consequently the fiber of the projection $\Omega(G \vee H) \rightarrow$ $\Omega H$ is

$$
\Omega\left(\bigvee_{n \geqslant 0} a d^{n}(H)(G)\right) .
$$

This is homotopy equivalent to $\Omega(G \rtimes \Omega H)$, and the map

$$
\bigvee_{n \geqslant 0} a d^{n}(H)(G) \rightarrow G \vee H
$$

which factors through $G \rtimes \Omega H$ establishes the homotopy equivalence in Theorem 3 ,

Corollary 3.5. (a) If $G$ is simply connected, $S \Omega G \simeq \bigvee_{n \geqslant 0} a d^{n}(G)(G)$,

(b) if both $G$ and $H$ are simply connected,

$$
\Omega G * \Omega H \simeq \bigvee_{\substack{i \geqslant 0 \\ j \geqslant 1}} a d^{j}(H)\left(a d^{i}(G)(G)\right) .
$$

Proof. For (a) apply Corollary 3.2 and Theorem 3 (a). For (b), we expand

$$
\begin{aligned}
\Omega G * \Omega H & \simeq(S \Omega G) \wedge \Omega H \\
& \simeq\left[\bigvee_{i \geqslant 0}\left(a d^{i}(G)(G)\right)\right] \wedge \Omega H \\
& \simeq \bigvee_{i \geqslant 0}\left[a d^{i}(G)(G) \wedge \Omega H\right] \\
& \simeq \bigvee_{\substack{i \geqslant 0 \\
j \geqslant 1}} a d^{j}(H)\left(a d^{i}(G)(G)\right)
\end{aligned}
$$

using Proposition 2.5 and Theorem 3(a).

Given a Theriault product $P=G_{1} \circ \cdots \circ G_{s}$ with some fixed association, let us write $\ell(P)=s$ for the length of $P$.

Theorem 3.6. Suppose $G$ and $H$ are both simply connected co- $H$ spaces and $k \geqslant 1$. Then there is a locally finite collection of iterated Theriault products $\left\{P_{\alpha}(k)\right\}$ of length $\ell_{\alpha}$ and iterated Whitehead product maps

$$
\omega_{\alpha}(k): P_{\alpha}(k) \rightarrow G \vee H
$$

such that

$$
\Omega(G \vee H) \simeq \Omega\left(\bigvee_{\ell_{\alpha}>k} P_{\alpha}(k)\right) \times \Omega\left(\prod_{\ell_{\alpha} \leqslant k} P_{\alpha}(k)\right),
$$

and the factors of the right-hand side are mapped to the left-hand side by the $\omega_{\alpha}(k)$.

Proof. For $k=1$ we use the decomposition

$$
\Omega(G \vee H) \simeq \Omega(\Omega G * \Omega H) \times \Omega(G \times H),
$$


where $\Omega G * \Omega H$ is a bouquet of iterated Theriault products of length at least 2 by Corollary 3.5(b). Now we proceed by induction on $k$. Among the finite list of products $P_{\alpha}(k)$ of length $k+1$, choose one which we label $P$. Then

$$
\begin{aligned}
\Omega\left(\bigvee_{\ell_{\alpha}>k} P_{\alpha}\right) & \simeq \Omega\left(P \vee \bigvee_{\substack{\ell_{\alpha}>k \\
P_{\alpha} \neq P}} P_{\alpha}\right) \\
& \simeq \Omega P \times \Omega\left(\bigvee_{\substack{\ell_{\alpha}>k \\
P_{\alpha} \neq P}} P_{\alpha} \rtimes \Omega P\right) .
\end{aligned}
$$

The second factor has one less product of length $k+1$. If we repeat this process once for each $P_{\alpha}(k)$ of length $k+1$, we obtain

$$
\Omega\left(\bigvee_{\ell\left(P_{\alpha}\right)>k} P_{\alpha}\right)=\Omega P_{j} \times \cdots \times \Omega P_{m} \times \Omega\left(\bigvee_{\ell\left(P_{\alpha}\right)>k+1} P_{\alpha}(k+1)\right)
$$

Now add the $P_{1} \cdots P_{m}$ to the list of $P_{\alpha}$ with $\ell\left(P_{\alpha}\right) \leq k$ to obtain all $P_{\alpha}(k+1)$ with length $\leq k+1$.

Corollary 3.7. Suppose $X$ is a finite dimensional co-associative co-H space and $f: X \rightarrow G \vee H$, where $G$ and $H$ are simply connected co- $H$ spaces. Then $f$ is a sum of iterated Whitehead products.

Proof. Suppose $\operatorname{dim} X=k$ and $f: X \rightarrow G \vee H$ is given. Decompose $\Omega(G \vee H)$ as in Theorem 3.6 and note that any product $P_{\alpha}$ of length $k$ is at least $k$ connected. Consequently the restriction of $\Omega f$,

$$
(\Omega X)^{k-1} \stackrel{\Omega f}{\longrightarrow} \Omega(G \vee H),
$$

factors through the product $\Omega\left(\prod_{\ell_{\alpha} \leq k} P_{\alpha}(k)\right)$ and the adjoint,

$$
S\left[(\Omega X)^{k-1}\right] \rightarrow G \vee H,
$$

is a sum of iterated Whitehead products. However, $f$ is the composition of this map with the $\mathrm{co}-\mathrm{H}$ space structure map

$$
X \rightarrow S\left[(\Omega X)^{k-1}\right]
$$

which is a co-H map, so $f$ is such a sum as well.

Proposition 3.8. If $G$ and $H$ are simply connected, then there is a homotopy equivalence

$$
\phi:(G \vee H) \cup_{W} C(G \circ H) \rightarrow G \times H .
$$

Proof. Since the composition

$$
G \circ H \rightarrow \Omega G * \Omega H \rightarrow G \vee H \rightarrow G \times H
$$

is null homotopic, there is an extension

$$
C=(G \vee H) \cup C(G \circ H) \stackrel{\phi}{\rightarrow} G \times H .
$$

The problem is to show that this map is a homotopy equivalence. We begin by observing that we can construct a right inverse $\zeta$ to $\Omega \phi$ as the sum of the loops on the inclusions of $G$ and $H$ into $C$ :

$$
\Omega G \times \Omega H \stackrel{\zeta}{\rightarrow} \Omega C \stackrel{\Omega \phi}{\longrightarrow} \Omega G \times \Omega H
$$


so $(\Omega \phi)_{*}: H_{*}(\Omega C) \rightarrow H_{*}(\Omega G \times \Omega H)$ is an epimorphism. We will complete the proof by showing that the rank of $H_{k}(\Omega C)$ is less than or equal to the rank of $H_{k}(\Omega G \times \Omega H)$. We will need several lemmas.

Lemma 3.9. Write $\Omega G * \Omega H \simeq G \circ H \vee S Q$. Then the restriction

$$
S Q \rightarrow \Omega G * \Omega H \stackrel{\omega}{\rightarrow} G \vee H \rightarrow C
$$

is null homotopic

Proof. We first look at the homotopy commutative diagram

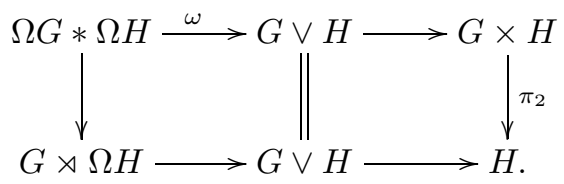

Applying Theorem 3(a) we see that the composition

$$
S Q \rightarrow \Omega G * \Omega H \rightarrow G \rtimes \Omega H \rightarrow G \vee H
$$

is a sum of maps $\gamma_{i}$ factoring through $a d^{i}(H)(G) \rightarrow G \vee H$ for $i \leqslant 2$. By induction on $i$ we see that

$$
a d^{i}(H)(G) \rightarrow G \vee H \rightarrow C
$$

is null homotopic for $i \geqslant 1$. This follows since $a d^{i}$ factors

$$
a d^{i}(H)(G) \rightarrow a d^{i-1}(H)(G) \vee H \stackrel{a d^{i-1} \vee 1}{\longrightarrow} G \vee H \vee H \rightarrow G \vee H \rightarrow C .
$$

It follows from Lemma 3.9 that the mapping cone of $\omega$ is homotopy equivalent to $C \vee S^{2} Q$. Recall that Ganea proved Gan70 that given a fibration sequence $F \rightarrow E \rightarrow B$, one can construct a fibration sequence

$$
F * \Omega B \rightarrow E \cup C F \stackrel{\pi}{\rightarrow} B,
$$

where $\pi$ pinches the cone on $F$ to a point. Apply this to the fibration sequence

$$
\Omega G * \Omega H \stackrel{\omega}{\rightarrow} G \vee H \rightarrow G \times H
$$

to obtain

$$
\Omega G * \Omega H * \Omega(G * H) \rightarrow C \vee S^{2} Q \stackrel{\pi}{\rightarrow} G \times H .
$$

It is possible that the map $\left.\pi\right|_{S^{2} Q}$ is nontrivial. However, $\left.\pi\right|_{S^{2} Q}$ is the sum of the projections onto $G$ and $H$, so it factors through $C$ up to homotopy. Using such a factorization we can construct a homotopy equivalence

$$
\Gamma: C \vee S^{2} Q \rightarrow C \vee S^{2} Q
$$

such that $\left.\pi \Gamma\right|_{S^{2} Q}$ is null homotopic. Replacing $\pi$ with $\pi \Gamma$ does not alter the homotopy type of the fiber of $\pi$, so we can form the following diagram of fibrations:

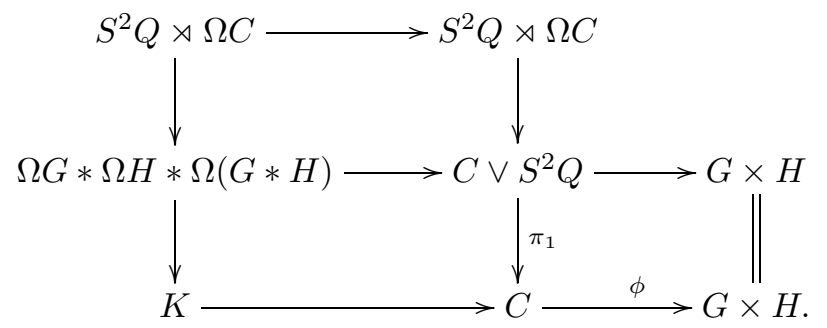


The left-hand verticle fibration has a cross section since $\pi_{1}$ does, hence $K$ is a co-H space and we have a splitting:

$$
\Omega(\Omega G * \Omega H * \Omega(G \times H)) \simeq \Omega\left(S^{2} Q \rtimes \Omega C\right) \times \Omega K .
$$

We now study the homology algebra with a field of coefficients. Each space here is the loop space on a co-H space, so all the homology algebras are tensor algebras. It follows that for each $i \geqslant 0$

$$
\operatorname{rank} H_{i}(\Omega G * \Omega H * \Omega(G \times H)) \geqslant \operatorname{rank} H_{i}\left(S^{2} Q \rtimes \Omega C\right) .
$$

Suppose now that we have two power series $f(t)=\Sigma a_{n} t^{n}$ and $g(t)=\Sigma b_{n} t^{n}$ with $a_{i}, b_{i}$ nonnegative integers. We will say that $f \geqslant g$ iff $a_{i} \geqslant b_{i}$ for each $i$. Write $\mathcal{X}(X)$ for the Poincaré series of a space $X$. In these terms, we have shown that

$$
\mathcal{X}(\Omega G * \Omega H * \Omega(G \times H)) \geqslant \mathcal{X}\left(S^{2} Q \rtimes \Omega C\right) .
$$

We now calculate the Poincaré series for each of these spaces. Suppose $\mathcal{X}(G)=1+g t$ and $\mathcal{X}(H)=1+h t$, where $g$ and $h$ are polynomial in $t$ with positive integral coefficient. We then have the following consequences:

$$
\begin{aligned}
\mathcal{X}(\Omega G) & =1+\frac{g}{1-g}, \\
\mathcal{X}(\Omega H) & =1+\frac{h}{1-h}, \\
\mathcal{X}(\Omega G * \Omega H) & =1+\frac{g h t}{(1-g)(1-h)}, \\
\mathcal{X}(G \circ H) & =1+g h t, \\
\mathcal{X}(S Q) & =1+g h t \frac{g+h-g h}{(1-g)(1-h)}, \\
\mathcal{X}\left(S^{2} Q \rtimes \Omega C\right) & =1+g h t^{2} \frac{g+h-g h}{(1-g)(1-h)} \cdot \mathcal{X}(\Omega C) .
\end{aligned}
$$

On the other hand,

$$
\mathcal{X}(\Omega G \times \Omega H)=1+\frac{g+h-g h}{(1-g)(1-h)} .
$$

Consequently,

$$
\mathcal{X}(\Omega G * \Omega H *(\Omega G \times \Omega H))=1+\frac{g h t^{2}(g+h-g h)}{(1-g)^{2}(1-h)^{2}},
$$

Observe that if $h(t)=\Sigma c_{n} t^{n} \neq 0$ also has nonnegative coefficients, $f(t) \geqslant g(t)$ iff $h(t) f(t) \geqslant h(t) g(t)$. It follows that $\mathcal{X}(\Omega C) \leq \frac{1}{(1-g)(1-h)}=\mathcal{X}(\Omega G \times \Omega H)$, so $\operatorname{rank} H_{i}(\Omega C) \leq \operatorname{rank} H_{i}(\Omega G \times \Omega H)$ for all $i$. Thus $(\Omega \phi)_{*}$ is an isomorphism, and hence $\phi_{*}$ is as well. Since this is true for any field of coefficients, $\phi_{*}$ induces isomorphisms in integral homology, and hence $\phi$ is a homotopy equivalence.

\section{REFERENCES}

[AG95] D. Anick and B. Gray, Small H spaces related to Moore spaces, Topology 34 (1995), no. 4, 859-881. MR1362790 (97a:55011)

[Ani93] D. Anick, Differential algebras in topology, Research Notes in Mathematics, vol. 3, A K Peters, Ltd., Wellesley, MA, 1993, xxxvi+274 pp. MR1213682(94h:55020) 
[Ark62] M. Arkowicz, The generalized Whitehead product, Pacific J. Math 12 (1962), no. 1, 7-23. MR0155328 (27:5262)

[CMN79] F. R. Cohen, J. C. Moore, and J. A. Neisendorfer, Torsion in homotopy groups, Ann. of Math. (2) 109 (1979), no. 1, 121-168. MR519355 (80e:55024)

[Coh57] D. E. Cohen, Products and carrier theory, Proc. London Math. Soc. 7 (1957), no. 3, 219-248. MR0087941 (19:441a)

[DT58] A. Dold and R. Thom, Quasifasserungen und unendliche symmetrische Producte, Ann. Math. 67 (1958), 239-281. MR0097062 (20:3542)

[Gan70] T. Ganea, Cogroups and suspensions, Invent. Math. 9 (1970), 185-197. MR0267582 $(42: 2484)$

[Gra71] B. Gray, A note on the Hilton-Milnor Theorem, Topology 10 (1971), 199-201. MR0281202 (43:6921)

[Gra88] , On the iterated suspension, Topology 27 (1988), no. 3, 301-310. MR963632 (89h:55016)

[GT10] B. Gray and S. Theriault, An elementary construction of Anick's fibration, Geometry and Topology 14 (2010), 243-276. MR2578305

[GTW] J. Grbić, S. Theriault, and J. Wu, Suspension splittings and James-Hopf Invariants for retracts of the loops on co-H spaces (private communication).

[Hil59] P. J. Hilton, Homotopy theory and duality, mimeographed notes, 1959, Cornell University.

[Nei09] J. Neisendorfer, Algebraic methods in unstable homotopy theory, New Mathematical Monographs, no. 12, Cambridge University Press, 2009. MR.2604913

[The03] S. D. Theriault, Homotopy decompositions involving the loops on a co-associative co-H space, Canada J. Math. 55 (2003), no. 1, 181-203. MR1952331(2003k:55006)

[Whi41] J. H. C. Whitehead, On adding relations to homotopy groups, Annals of Math. 42 (1941), no. 2, 409-428. MR0004123 (2:323c)

Department of Mathematics, Statistics and Computer Science, University of Illinois at Chicago, 851 S. Morgan Street, Chicago, Illinois 60607-7045

E-mail address: brayton@uic.edu 